\title{
Multistep Prediction using Point-Cloud Approximation of Continuous Belief Functions
}

\author{
Thierry Denoeux \\ Université de Technologie de Compiègne, CNRS \\ UMR 7253 Heudiasyc, Compiègne, France \\ Email: tdenoeux@utc.fr
}

\author{
Orakanya Kanjanatarakul \\ Chiang Mai Rajabhat University \\ Chiang Mai, Thailand \\ Email: orakanyaa@gmail.com
}

\begin{abstract}
We consider the problem of quantifying prediction uncertainty in the Dempster-Shafer framework. Our approach assumes a parametric statistical model relating the variable of interest, the parameter and a pivotal random variable with known probability distribution. A predictive belief function is computed using this model and a belief function defined in the parameter space. In the case of multistep prediction, the quantity to be predicted is a vector, and the predictive belief function is defined in a multidimensional space, making its representation and manipulation difficult. To address this issue, we propose to approximate the focal sets of belief functions using point clouds, which allows us to approximate the belief and plausibility of arbitrary events with any accuracy. As an illustration, the approach is applied to the case of a first-order autoregressive process.

Index Terms-Dempster-Shafer theory, evidence theory, imprecise probabilities, possibility measure, forecasting, point clouds, uncertainty quantification, autoregressive process.
\end{abstract}

\section{INTRODUCTION}

In many areas, it is important to guess the values of some not yet observed variables in order to support decision-making. A good prediction system not only determines the quantities of interest with low error most of the time, but it is also capable of quantifying the uncertainty of the predictions. In previous work [1], [2], we have argued for the Dempster-Shafer theory of belief functions [3], [4] as a suitable framework for quantifying prediction uncertainty. The approach introduced in [1] postulates the existence of a parametric statistical model describing the data generating process. Such a model typically allows us to describe the variable $Y$ of interest as a function of some parameter $\theta$ and a random variable $U$ with known distribution. For instance, if $Y$ has a normal distribution with mean $\mu$ and standard deviation $\sigma$, it can be written as

$$
Y=\mu+\sigma \Phi^{-1}(U)=\varphi(\theta, U),
$$

where $\theta=(\mu, \sigma), \Phi$ is the standard normal cumulative distribution function (cdf) and $U$ has a standard uniform distribution in $[0,1]$. An equation such as (1) will be referred to as a $\varphi$-equation. Typically, parameter $\theta$ is unknown, but a belief function on $\theta$ can be constructed from past observations using the likelihood function [4], [5]. This belief function, together

This research was supported by the Labex MS2T, which was funded by the French Government, through the program "Investments for the future" by the National Agency for Research (reference ANR-11-IDEX-0004-02). with the probability distribution of $U$, can be propagated through the $\varphi$-equation to compute a belief function $\mathrm{Bel}_{Y}$ on $Y$.

In [1] and [2], we have described numerical schemes allowing us to approximate $\mathrm{Bel}_{Y}$ through Monte Carlo simulation and constrained optimization. These schemes, however, can only be used when $Y$ is a scalar quantity and the focal sets of $\mathrm{Bel}_{Y}$ are real intervals. In some problems such as multistep time series forecasting, $Y$ is an $h$-dimensional random vector, with $h>1$. The belief function $\operatorname{Bel}_{Y}$ is then still well defined mathematically, but its focal sets are regions of $\mathbb{R}^{h}$ with arbitrary shape. Computing the degree of belief $\operatorname{Bel}_{Y}(B)$ of events $B$ of interest then becomes more challenging. In this paper, we propose to tackle this problem by combining Monte Carlo simulation and a representation of focal sets using point clouds.

The rest of this paper is organized as follows. The necessary background on belief functions will first be recalled in Section II. The application to prediction and the proposed scheme for approximating a multidimensional predictive belief function will then be described in Section III. Finally, as an illustration, our approach will be applied to multistep time series prediction with an $A R(1)$ model in Section IV, and concluding remarks will be given in Section V.

\section{BELIEF FUNCTIONS}

To make the paper self-contained, the basic notions of belief and plausibility functions will first be recalled in Section II-A. The relation with random sets, which is instrumental in our approach, will then be explained in Section II-B, and the special case of consonant belief functions will be addressed in Section II-C. Finally, Dempster's rule will be recalled in Section II-D.

\section{A. Definitions}

Let $\Omega$ be a set (not necessarily finite) and $\mathcal{B}$ an algebra of subsets of $\Omega$ (i.e., a nonempty family of subsets of $\Omega$, closed under complementation and finite intersection). A belief function on $\mathcal{B}$ is a mapping $\mathrm{Bel}: \mathcal{B} \rightarrow[0,1]$ verifying $\operatorname{Bel}(\emptyset)=0, \operatorname{Bel}(\Omega)=1$ and the complete monotonicity 
property: for any $k \geq 2$ and any collection $B_{1}, \ldots, B_{k}$ of elements of $\mathcal{B}$,

$$
\operatorname{Bel}\left(\bigcup_{i=1}^{k} B_{i}\right) \geq \sum_{\emptyset \neq I \subseteq\{1, \ldots, k\}}(-1)^{|I|+1} \operatorname{Bel}\left(\bigcap_{i \in I} B_{i}\right) .
$$

A function $P l: \mathcal{B} \rightarrow[0,1]$ is a plausibility function iff the mapping $B \rightarrow 1-P l(\bar{B})$ is a belief function. In DempsterShafer theory [4], belief functions are used to represent an agent's belief about some unknown quantity $Y$ taking values in $\Omega$, based on some evidence. The number $\operatorname{Bel}(B)$ for any event $B \in \mathcal{B}$ is interpreted as the total degree of support in $B$, while $P l(B)$ measures the lack of support in the complement of $B$. Complete ignorance is, thus, represented by the vacuous belief function verifying $\operatorname{Bel}(B)=0$ for all $B \neq \Omega$, and $P l(B)=1$ for all $B \neq \emptyset$.

\section{B. Random set representation}

A belief function can always be induced by a random set [3], [6], [7]. This point of view is particularly useful to define and manipulate belief functions on continuous spaces such as $\mathbb{R}^{p}$ [2]. More precisely, let $S$ be a state space, $\mathcal{A}$ an algebra of subsets of $S$, and $\mathbb{P}$ a finitely additive probability on $(S, \mathcal{A})$. A mapping $\Gamma$ from $S$ to $2^{\Omega}$ (referred to as a multivalued mapping) is said to be strongly measurable with respect to $(S, \mathcal{A})$ and $(\Omega, \mathcal{B})$ if, for any $B \in \mathcal{B}$, the set

$$
\Gamma_{*}(B)=\{s \in S \mid \Gamma(s) \neq \emptyset, \Gamma(s) \subseteq B\}
$$

belongs to $\mathcal{A}$. Then, the mapping $\mathbb{P}_{*}$ from $\mathcal{B}$ to $[0,1]$ defined as

$$
\mathbb{P}_{*}(B)=\frac{\mathbb{P}\left(\Gamma_{*}(B)\right)}{\mathbb{P}\left(\Gamma_{*}(\Omega)\right)},
$$

for all $B \in \mathcal{B}$, is a belief function [6]. The sets $\Gamma(s)$ are called the focal sets of $\mathbb{P}_{*}$.

\section{Consonant belief functions}

The random-set view of belief functions outlined in the previous section provides a way to define practical models using well-chosen probability spaces $(S, \mathcal{A}, \mathbb{P})$ and multivalued mappings $\Gamma$. In particular, let $S$ be the interval $[0,1], \mathcal{A}=\beta_{[0,1]}$ the Borel $\sigma$-field on $S, \mathbb{P}=\lambda$ the Lebesgue measure on $[0,1]$, and $\pi$ a mapping from $\Omega$ to $S$ s.t. $\sup _{\omega \in \Omega} \pi(\omega)=1$. Then, the mapping $\Gamma: S \mapsto 2^{\Omega}$ defined by

$$
\Gamma(s)=\{\omega \in \Omega \mid \pi(\omega) \geq s\}
$$

for all $s \in[0,1]$ is strongly measurable [8]. In this case, the induced belief function is consonant, i.e., for any $s$ and $s^{\prime}$ in $[0,1]$, we have either $\Gamma(s) \subseteq \Gamma\left(s^{\prime}\right)$ or $\Gamma\left(s^{\prime}\right) \subseteq \Gamma(s)$, and the corresponding plausibility function is a possibility measure [9]; it can be computed as $P l(B)=\sup _{\omega \in B} \pi(\omega)$ for all $B \subseteq \Omega$. In particular, $\operatorname{Pl}(\{\theta\})=\pi(\theta)$. (Function $\pi$ is a possibility distribution; it is often referred to in this context as the contour function). This model is useful, for instance, in statistical inference, where $\pi$ can be taken to be the relative likelihood function [4], [5], yielding a consonant belief function on the parameter space.

\section{Dempster's rule}

Dempster-Shafer theory is fundamentally based on the idea of combining belief functions induced by independent pieces of evidence [3], [4], [10]. Given two belief functions $B e l_{1}$ and $\mathrm{Bel}_{2}$ on $\Omega$ generated by random sets $\left(S_{i}, \mathcal{A}_{i}, \mathbb{P}_{i}, \Gamma_{i}\right), i=1,2$, the orthogonal sum of $\mathrm{Bel}_{1}$ and $\mathrm{Bel}_{2}$, denoted by $\mathrm{Bel}_{1} \oplus$ $\mathrm{Bel}_{2}$, is the belief function generated by the random set $\left(S_{1} \times\right.$ $\left.S_{2}, \mathcal{A}_{1} \otimes \mathcal{A}_{2}, \mathbb{P}_{1} \otimes \mathbb{P}_{2}, \Gamma_{\cap}\right)$, where $\mathcal{A}_{1} \otimes \mathcal{A}_{2}$ is the algebra generated by sets $A_{1} \times A_{2}$, with $A_{1} \in \mathcal{A}_{1}$ and $A_{2} \in \mathcal{A}_{2}$, $\mathbb{P}_{1} \otimes \mathbb{P}_{2}$ is the product of $\mathbb{P}_{1}$ and $\mathbb{P}_{2}$, and

$$
\Gamma_{\cap}\left(s_{1}, s_{2}\right)=\Gamma_{1}\left(s_{1}\right) \cap \Gamma_{2}\left(s_{2}\right) .
$$

Practically, Dempster's rule often needs to be approximated by Monte Carlo simulation [2], [11]. Typically, we draw pairs $\left(s_{1}, s_{2}\right)$ from $\mathbb{P}_{1} \otimes \mathbb{P}_{2}$ and keep them only if $\Gamma_{\cap}\left(s_{1}, s_{2}\right) \neq \emptyset$. After obtaining $N$ such pairs $\left(s_{1}^{(1)}, s_{2}^{(1)}\right), \ldots,\left(s_{1}^{(N)}, s_{2}^{(N)}\right)$, we get an approximate representation of $\mathrm{Bel}_{1} \oplus \mathrm{Bel}_{2}$ as $N$ nonempty focal sets $\Gamma_{\cap}\left(s_{1}^{(i)}, s_{2}^{(i)}\right), i=1, \ldots, N$. The degree of belief $\operatorname{Bel}_{12}(B)=\left(\operatorname{Bel}_{1} \oplus \operatorname{Bel}_{2}\right)(B)$ in any event $B$ is then approximated by

$$
\widehat{B e l}_{12}(B)=\frac{1}{N} \sum_{i=1}^{N} I\left(\Gamma_{\cap}\left(s_{1}^{(i)}, s_{2}^{(i)}\right) \subseteq B\right)
$$

where $I(\cdot)$ is the indicator function.

\section{APPLICATION TO PREDICTION}

From now on, we focus on the application of belief functions to statistical prediction. The prediction of a scalar quantity, as required for one-step-ahead prediction, will first be addressed in Section III-A. Multistep prediction will then be tackled in Section III-B.

\section{A. One-step-ahead prediction}

We consider a random vector $\boldsymbol{X}=\left(X_{1}, \ldots, X_{T}, X_{T+1}\right) \in$ $\mathcal{X}^{T+1}$ whose joint distribution depends on some unknown parameter $\theta \in \Theta$. Let $\boldsymbol{X}_{1: T}$ be the vector containing the first $T$ components of $\boldsymbol{X}$. Assume that we have observed $\boldsymbol{X}_{1: T}=\boldsymbol{x}_{1: T}$ and we want to predict $X_{T+1}$. The approach introduced in [1] is to predict a random variable $Y$ with the same probability distribution as $X_{T+1}$ given $\boldsymbol{X}_{1: T}=\boldsymbol{x}_{1: T}$, of the form

$$
Y=\varphi_{\boldsymbol{x}_{1: T}}(\theta, U)
$$

where $U$ is a random variable with known probability distribution, and $\varphi_{\boldsymbol{x}_{1: T}}$ is a mapping from $\Theta \times \mathbb{R}$ to $\mathbb{R}$ depending on $\boldsymbol{x}_{1: T}$. In particular, we can define $\varphi_{\boldsymbol{x}_{1: T}}$ as

$$
\varphi_{\boldsymbol{x}_{1: T}}(\theta, U)=F_{Y \mid \boldsymbol{X}_{1: T}=\boldsymbol{x}_{1: T}}^{-1}(U ; \theta),
$$

where $F_{Y \mid \boldsymbol{X}_{1: T}=\boldsymbol{x}_{1: T}}^{-1}(\cdot ; \theta)$ is the inverse (or the generalized inverse if $Y$ is discrete) of the cumulative distribution function of $Y$ given $\boldsymbol{X}_{1: T}=\boldsymbol{x}_{1: T}$, and $U$ has a standard uniform distribution in $[0,1]$.

In (3), random variable $U$ accounts for random uncertainty, while lack of knowledge of $\theta$ corresponds to epistemic uncertainty. This latter uncertainty can be represented by a belief 
function $\mathrm{Bel}_{\theta}$, which can be propagated, together with the pivotal random variable $U$, through (3) to obtain a belief function on $Y$ or, equivalently, on $X_{T+1}$. A belief $\mathrm{Bel}_{\theta}$ on $\theta$ can be defined from the likelihood function, or from a confidence region. These two methods are briefly recalled below.

a) Likelihood-based belief function: In [1], [2], it was proposed to consider the likelihood-based belief function, defined as the consonant belief function $B e l_{\theta}$ whose contour function is the relative likelihood function $p l$ defined as

$$
\pi(\theta)=p l(\theta)=\frac{L\left(\theta ; \boldsymbol{x}_{1: T}\right)}{L\left(\widehat{\theta} ; \boldsymbol{x}_{1: T}\right)},
$$

where $L$ denotes the likelihood function, $\widehat{\theta}$ the maximum likelihood estimate (MLE) of $\theta$, and it is assumed that $L\left(\widehat{\theta} ; \boldsymbol{x}_{1: T}\right)<\infty$. Combining $\mathrm{Bel}_{\theta}$ with a Bayesian prior on $\theta$ then yields the Bayesian posterior, ensuring compatibility with Bayesian inference when a probabilistic prior is available.

b) Confidence belief function: Alternatively, it was proposed in [12] to model our beliefs in $\theta$ using the logical belief function $\mathrm{Bel}_{\theta}$ such that $\operatorname{Bel}_{\theta}(B)=I\left(R_{1-\alpha} \subseteq B\right)$, where $R_{1-\alpha}$ is a $100(1-\alpha) \%$ confidence region on $\theta$. The only focal set of $\mathrm{Bel}_{\theta}$ is then $R_{1-\alpha}$, and the corresponding contour function is the indicator function of $R_{1-\alpha}: \pi(\theta)=I\left(\theta \in R_{1-\alpha}\right)$ for all $\theta \in \Theta$. When propagating such a confidence belief function in (3), we get a calibrated predictive belief function $\mathrm{Bel}_{Y}$ that is dominated by the true conditional distribution of $Y$ given $\boldsymbol{X}_{1: T}=\boldsymbol{x}_{1: T}$ for $100(1-\alpha) \%$ of the samples $\boldsymbol{x}_{1: T}$ [12]. Under regularity conditions, an approximate $1-\alpha$ confidence region can be obtained from the relative likelihood function as

$$
R_{1-\alpha}=\left\{\theta \in \Theta \mid p l(\theta) \geq \exp \left(-0.5 \chi_{p ; 1-\alpha}^{2}\right)\right\}
$$

where $\chi_{p ; 1-\alpha}^{2}$ is the $1-\alpha$ quantile of the chi square distribution with $p$ degrees of freedom, and $p$ is the dimension of $\theta$ [13].

The propagation of $\operatorname{Bel}_{\theta}$ and the probability measure $\mathbb{P}_{U}$ of random variable $U$ through (3) can be formalized as a combination by Dempster's rule [2]. It can be approximated using the Monte Carlo approach described in Section II-D. The belief function $\mathrm{Bel}_{\theta}$ on $\theta$ constructed by any of the two methods mentioned above is induced by a random set $\left([0,1], \lambda, \beta_{[0,1]}, \Gamma\right)$, where $\Gamma$ is defined by $\Gamma(s)=\{\theta \in \Theta \mid$ $\pi(\theta) \geq s\}$. The corresponding predictive belief function on $Y$ is induced by the multi-valued mapping $\Lambda$ defined by

$$
\Lambda:(s, u) \mapsto \Lambda(s, u)=\varphi_{\boldsymbol{x}_{1: T}}(\Gamma(s), u) .
$$

Assuming, without loss of generality, that $U$ has a standard uniform distribution, a Monte Carlo simulation approach can be implemented by picking $N$ pairs $\left(s_{i}, u_{i}\right), i=1, \ldots, N$ uniformly in $[0,1]^{2}$ and approximating the predictive belief function $\operatorname{Bel}_{Y}$ by the $N$ focal sets $\Lambda\left(s_{i}, u_{i}\right)$. If these sets are real intervals, their bounds can be found by searching for the minimum and the maximum of $\varphi_{\boldsymbol{x}_{1: T}}\left(\theta, u_{i}\right)$ under the constraint $\pi(\theta) \geq s_{i}$ (see [1], [2]). This approach will be illustrated in Section IV-A.

\section{B. Multiple-step-ahead prediction}

Let us now consider a random vector

$$
\boldsymbol{X}=\left(X_{1}, \ldots, X_{T}, X_{T+1}, \ldots, X_{T+h}\right) \in \mathcal{X}^{T+h} .
$$

We assume that the first $T$ components have been observed: $\boldsymbol{X}_{1: T}=\boldsymbol{x}_{1: T}$, and the task is to predict the next $h$ components $\boldsymbol{X}_{T+1: T+h}$, which have not yet been observed. As before, we can define a random vector $\boldsymbol{Y}$ with the same distribution as $\boldsymbol{X}_{T+1: T+h}$ given $\boldsymbol{X}_{1: T}=\boldsymbol{x}_{1: T}$, of the form

$$
\boldsymbol{Y}=\varphi_{\boldsymbol{x}_{1: T}}(\theta, \boldsymbol{U}),
$$

where $\boldsymbol{U}$ is random vector with known distribution, and propagate a belief function on $\Theta$, together with $\mathbb{P}_{\boldsymbol{U}}$, through (5) (see details in [2]). However, the focal sets of the resulting predictive belief function $\operatorname{Bel}_{\boldsymbol{Y}}$ are now regions of $\mathbb{R}^{h}$, for which we need to find a simple and workable representation. In this paper, we propose a solution of this problem based on the approximation of the focal sets of $\mathrm{Bel}_{\theta}$ by finite sets of points, and their propagation in the $\varphi$-equation.

Assume that $\Theta$ is a bounded region of $\mathbb{R}^{p}$. (If $\Theta$ is not bounded, it is always possible to restrict it to a bounded region containing all parameter values $\theta$ with relative likelihood $p l(\theta)$ exceeding some threshold $\epsilon>0$ ). Let $\widetilde{\Theta}=\left\{\theta_{1}, \ldots, \theta_{M}\right\} \subset \Theta$ be a point cloud [14], defined as a set of $M$ points generated uniformly in $\Theta$. To generate the elements of $\widetilde{\Theta}$, we can use a pseudo-random number generator, but a more homogeneous covering of $\Theta$ is achieved by using a quasi-random lowdiscrepancy sequence such as a Halton sequence [14], [15]. As before, assume that $\mathrm{Bel}_{\theta}$ is induced by a random set $\left([0,1], \lambda, \beta_{[0,1]}, \Gamma\right)$, where $\Gamma$ is defined by $\Gamma(s)=\{\theta \in \Theta \mid$ $\pi(\theta) \geq s\}$. A focal set $\Gamma(s)$ of $\operatorname{Bel}_{\theta}$ can be approximated by the set $\widetilde{\Gamma}(s)$ containing the points $\theta$ in $\widetilde{\Theta}$ whose plausibility $\pi(\theta)$ is greater than $s$ :

$$
\widetilde{\Gamma}(s)=\{\theta \in \widetilde{\Theta} \mid \pi(\theta) \geq s\} .
$$

As set $\widetilde{\Gamma}(s)$ is finite, it is straightforward to propagate it through the $\varphi$-equation (5) by computing $\varphi_{\boldsymbol{x}_{1: T}}(\theta, \boldsymbol{u})$ for each $\theta \in \widetilde{\Gamma}(s)$. A focal set $\Lambda(s, \boldsymbol{u})=\varphi_{\boldsymbol{x}_{1: T}}(\Gamma(s), \boldsymbol{u})$ of $\mathrm{Bel}_{\boldsymbol{Y}}$ can then be approximated by

$$
\widetilde{\Lambda}(s, \boldsymbol{u})=\varphi_{\boldsymbol{x}_{1: T}}(\widetilde{\Gamma}(s), \boldsymbol{u})=\left\{\varphi_{\boldsymbol{x}_{1: T}}(\theta, \boldsymbol{u}) \mid \theta \in \widetilde{\Gamma}(s)\right\} .
$$

Using this scheme, the predictive belief function $\mathrm{Bel}_{\boldsymbol{Y}}$ can be approximated by drawing $N$ pairs $\left(s_{i}, \boldsymbol{u}_{i}\right)$ and determining the focal sets $\widetilde{\Lambda}\left(s_{i}, \boldsymbol{u}_{i}\right)$. For any subset $B$ of $\mathcal{X}^{h}$, the degree of belief and plausibility of $B$ can be approximated, respectively, by

$$
\widehat{\operatorname{Bel}}(B)=\frac{1}{N} \sum_{i=1}^{N} I\left(\widetilde{\Lambda}\left(s_{i}, \boldsymbol{u}_{i}\right) \subseteq B\right)
$$

and

$$
\widehat{P l}(B)=\frac{1}{N} \sum_{i=1}^{N} I\left(\widetilde{\Lambda}\left(s_{i}, \boldsymbol{u}_{i}\right) \cap B \neq \emptyset\right) .
$$

In the next section, we illustrate this method in the special case of a first order autoregressive model. 


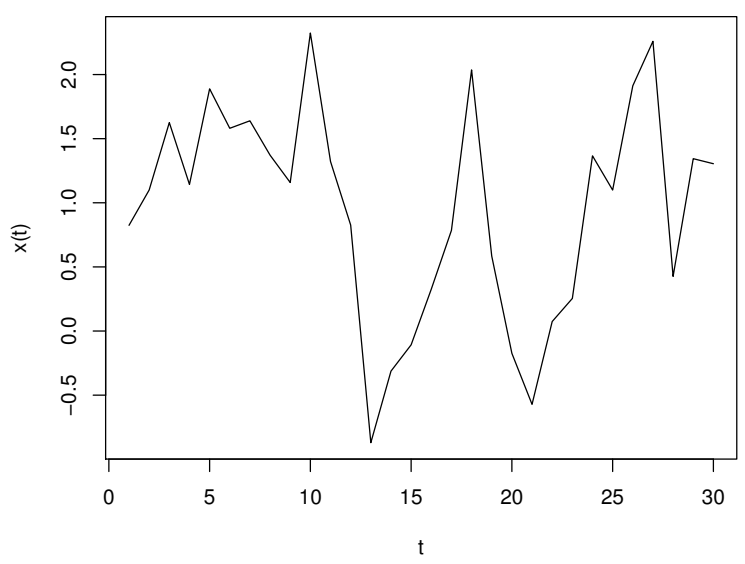

Fig. 1. Synthetic AR(1) time series.

\section{Application to The AR(1) MODEL}

Autoregressive models (AR) are commonly used to describe time-varying phenomena in many areas such as, e.g., economics [16] or hydrology [17]. In this section, we consider, as an illustration, a zero-mean $\mathrm{AR}(1)$ process governed by the equation

$$
X_{t}=\rho X_{t-1}+\epsilon_{t}, \quad t=1,2, \ldots,
$$

where $\rho \in(-1,1)$ is a parameter and $\epsilon_{t} \sim \mathcal{N}\left(0, \sigma^{2}\right)$ is a Gaussian error term with zero mean and standard deviation $\sigma$. Figure 1 shows an example of a synthetic time series of length $T=30$ generated from this model with $\rho=0.7$ and $\sigma=1$.

Given that the marginal distribution of $X_{1}$ is

$$
X_{1} \sim \mathcal{N}\left(0, \frac{\sigma^{2}}{1-\rho^{2}}\right)
$$

and the conditional distribution of $X_{t}$ given $X_{t-1}=x_{t-1}$ is $X_{t} \mid x_{t-1} \sim \mathcal{N}\left(\rho x_{t-1}, \sigma^{2}\right)$, the likelihood function after observing the first $T$ terms of the sequence can easily be computed as

$$
L\left(\theta ; \boldsymbol{x}_{1: T}\right)=\phi\left(x_{1} ; 0, \frac{\sigma}{\sqrt{1-\rho^{2}}}\right) \prod_{t=2}^{T} \phi\left(x_{t} ; \rho x_{t-1}, \sigma\right),
$$

where $\theta=(\rho, \sigma)$ and $\phi(\cdot ; \mu, \sigma)$ is the normal probability density function with mean $\mu$ and standard deviation $\sigma$. The likelihood-based contour function is then $p l(\theta)=$ $L\left(\theta ; \boldsymbol{x}_{1: T}\right) / L\left(\widehat{\theta} ; \boldsymbol{x}_{1: T}\right)$, where the MLE $\widehat{\theta}$ has to be computed numerically. Figure 2 shows contours of function $p l(\theta)$ for the data of Figure 1.

One-step and multiple-step ahead prediction with this model will be addressed, respectively, in Sections IV-A and IV-B.

\section{A. One-step ahead prediction}

To predict $X_{T+1}$, having observed $\boldsymbol{X}_{1: T}=\boldsymbol{x}_{1: T}$, we introduce the following $\varphi$-equation:

$$
Y=\rho x_{T}+\sigma \Phi^{-1}(U)=\varphi_{\boldsymbol{x}_{1: T}}(\theta, U),
$$

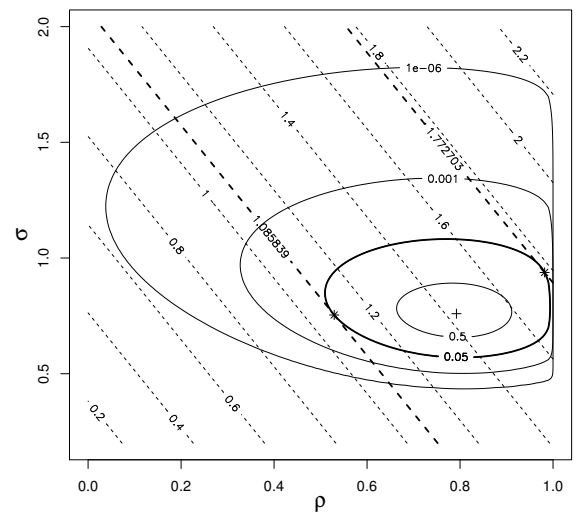

Fig. 2. Contours of the relative likelihood function $p l(\theta)$ (solid lines) and

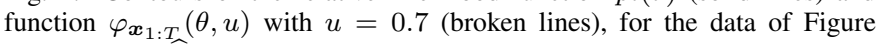
1. The MLE $\hat{\theta}$ of $\theta$ is shown as a + . The minimum and maximum of $\varphi_{\boldsymbol{x}_{1: T}}(\theta, 0.7)$ subject to $p l(\theta) \geq 0.05$ correspond to the bold broken lines, and the corresponding values of $\theta$ are shown as $*$.

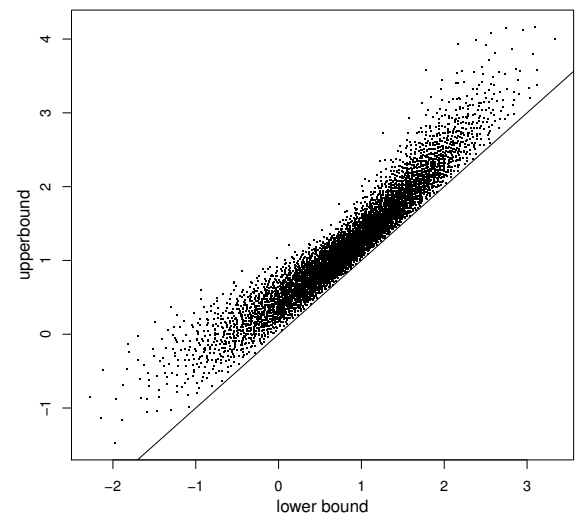

Fig. 3. Lower vs. upper bounds for $N=5000$ focal intervals $\left[y_{i}^{-}, y_{i}^{+}\right]$ generated by picking 5000 pairs $\left(s_{i}, u_{i}\right)$ randomly in $[0,1]^{2}$.

where $Y \sim \mathcal{N}\left(\rho x_{T}, \sigma^{2}\right)$ has the same distribution as $X_{T+1}$ given $\boldsymbol{X}_{1: T}=\boldsymbol{x}_{1: T}$ and $U$ has a standard uniform distribution.

Figure 2 shows the contour lines of functions $p l(\theta)$ and $\varphi_{\boldsymbol{x}_{1: T}}(\theta, u)$ for $u=0.7$ and the data of Figure 1. The bounds of the focal interval $\left[y_{i}^{-}, y_{i}^{+}\right]=\varphi_{\boldsymbol{x}_{1: T}}\left(\Gamma\left(s_{i}\right), u_{i}\right)$ for a given pair $\left(s_{i}, u_{i}\right)$ are found by searching for the minimum and the maximum of $\varphi_{\boldsymbol{x}_{1: T}}\left(\theta, u_{i}\right)$ subject to $p l(\theta) \geq s_{i}$. As shown in Figure 2, for $u_{i}=0.7$ and $s_{i}=0.05$ we get $\left[y_{i}^{-}, y_{i}^{+}\right]=[1.09,1.77]$. Figure 3 displays $N=5000$ such intervals $\left[y_{i}^{-}, y_{i}^{+}\right]$generated by picking 5000 pairs $\left(s_{i}, u_{i}\right)$ randomly in $[0,1]^{2}$. These focal intervals are a discrete representation of the predictive belief function $\mathrm{Bel}_{Y}$. For instance, the empirical cdfs of the upper and lower bounds of intervals $\left[y_{i}^{-}, y_{i}^{+}\right]$approximate the lower and upper cdfs of $B_{e} l_{Y}$ defined, respectively, as functions $y \mapsto \operatorname{Bel}((-\infty, y])$ and $y \mapsto P l((-\infty, y])$ (see Figure 4). 


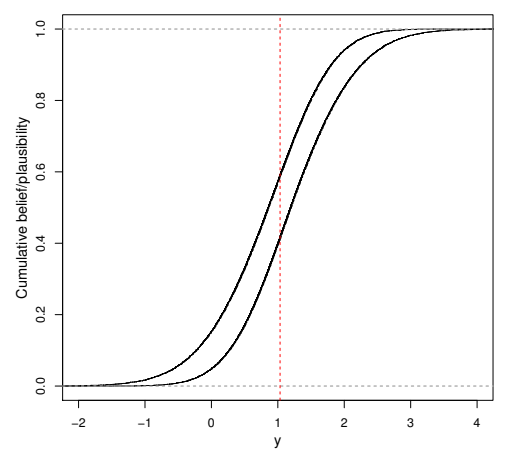

Fig. 4. Approximations of the lower and upper cdfs of $\mathrm{Bel}_{Y}$ computed using the 5000 focal intervals $\left[y_{i}^{-}, y_{i}^{+}\right]$displayed in Figure 3 . The vertical broken line corresponds to the plug-in prediction $\widehat{x}_{T+1}=\widehat{\rho} x_{T}$.

\section{B. Multistep prediction}

We now consider multistep prediction. For instance, for $h=$ 3 steps ahead, we have

$$
\begin{aligned}
& x_{T+1}=\rho x_{T}+\epsilon_{T+1} \\
& x_{T+2}=\rho x_{T+1}+\epsilon_{T+2} \\
& x_{T+3}=\rho x_{T+2}+\epsilon_{T+3},
\end{aligned}
$$

where $\epsilon_{T+1}, \epsilon_{T+2}$ and $\epsilon_{T+2}$ are independent random variables with normal distribution $\mathcal{N}\left(0, \sigma^{2}\right)$. Given $X_{T}=x_{T}$, the random vector $\boldsymbol{X}_{T+1: T+3}=\left(X_{T+1}, X_{T+2}, X_{T+3}\right)^{\prime}$ has the same distribution as

$$
\boldsymbol{Y}=\varphi_{\boldsymbol{x}_{1: T}}(\theta, \boldsymbol{U})=\left(Y_{1}, Y_{2}, Y_{3}\right)^{\prime}
$$

with

$$
\begin{aligned}
& Y_{1}=\rho x_{T}+\sigma \Phi^{-1}\left(U_{1}\right) \\
& Y_{2}=\rho Y_{1}+\sigma \Phi^{-1}\left(U_{2}\right) \\
& Y_{3}=\rho Y_{2}+\sigma \Phi^{-1}\left(U_{3}\right),
\end{aligned}
$$

where $\boldsymbol{U}=\left(U_{1}, U_{2}, U_{3}\right)$, and $U_{1}, U_{2}$ and $U_{3}$ are independent random variables with a standard uniform distribution $\mathcal{U}([0,1])$.

As explained in Section III-B, the focal sets $\Lambda(s, \boldsymbol{u})=$ $\varphi_{\boldsymbol{x}_{1: T}}(\Gamma(s), \boldsymbol{u})$ of $\mathrm{Bel}_{\boldsymbol{Y}}$ are now regions of $\mathbb{R}^{3}$, which will be represented by finite sets of points. For that purpose, we start by representing the parameter space $\Theta$ by a set $\widetilde{\Theta}$ of $M=3 \times 10^{4}$ points, as shown in Figure 5a. A focal set $\Gamma(s)=\{\theta \in \Theta \mid p l(\theta) \geq s\}$ of $\mathrm{Bel}_{\theta}$ is then represented by a finite set of points $\widetilde{\Gamma}(s)=\{\theta \in \widetilde{\Theta} \mid \pi(\theta) \geq s\}$ (see Figure $5 b)$. For any realization $\boldsymbol{u}$ of $\boldsymbol{U}$, we can then approximate the focal set $\Lambda(s, \boldsymbol{u})$ by computing the image $\varphi_{\boldsymbol{x}_{1: T}}(\theta, \boldsymbol{u})$ of each $\theta \in \widetilde{\Gamma}(s)$. We then obtain the set $\widetilde{\Lambda}(s, \boldsymbol{u})=\varphi_{\boldsymbol{x}_{1: T}}(\widetilde{\Gamma}(s), \boldsymbol{u})$. The two-dimensional projections of $\Lambda(s, \boldsymbol{u})$ on the planes spanned by $\left(Y_{1}, Y_{2}\right)$ and $\left(Y_{2}, Y_{3}\right)$ for three different randomly picked values of $\boldsymbol{u}$ are shown, respectively, in Figures $5 \mathrm{c}$ and $5 \mathrm{~d}$. The convex hulls of 100 focal sets $\widetilde{\Lambda}\left(s_{i}, \boldsymbol{u}_{i}\right)$ are shown in Figure 6.

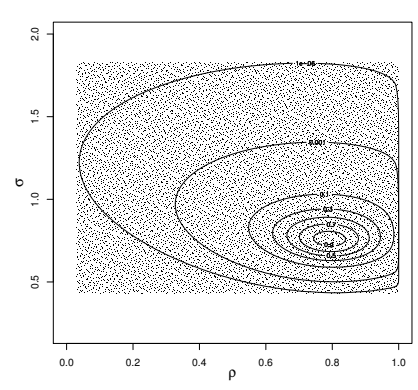

(a) $\widetilde{\Theta}$

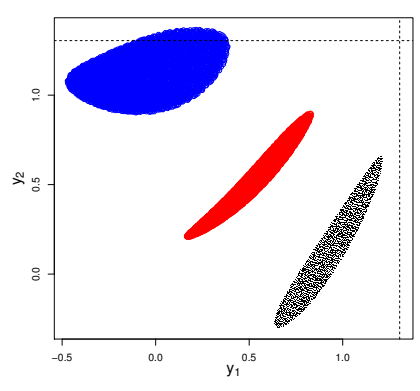

(c) $[\widetilde{\Lambda}(s, \boldsymbol{u})]_{12}$

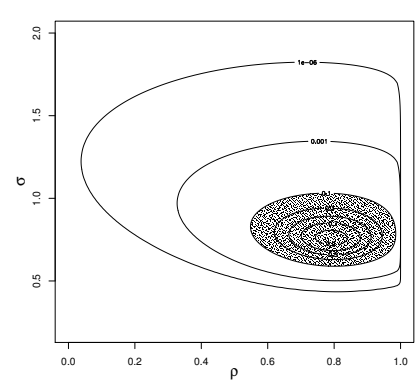

(b) $\widetilde{\Gamma}(s)$

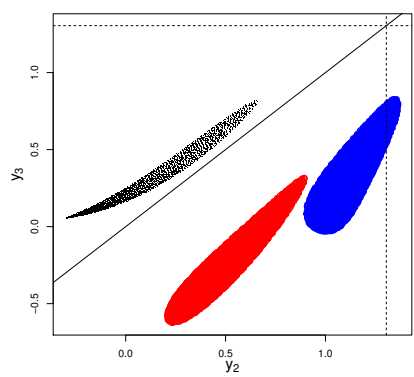

(d) $[\widetilde{\Lambda}(s, \boldsymbol{u})]_{23}$
Fig. 5. Illustration of the approximation of focal sets using point clouds. (a): Approximation of parameter space $\Theta$ by a finite set $\widetilde{\Theta}$ of $M$ points; (b): Approximation of a focal set $\Gamma(s)$ of $\mathrm{Bel}_{\theta}$ for $s=0.1$; (c) and (d): 2-D projections of focal sets $\widetilde{\Lambda}(s, \boldsymbol{u})$ for $s=0.1$ and three different values of $\boldsymbol{u}$.

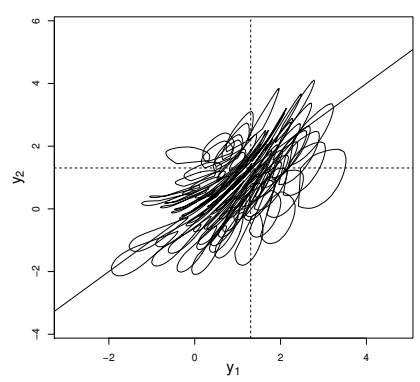

(a) $\left[\widetilde{\Lambda}\left(s_{i}, \boldsymbol{u}_{i}\right)\right]_{12}$

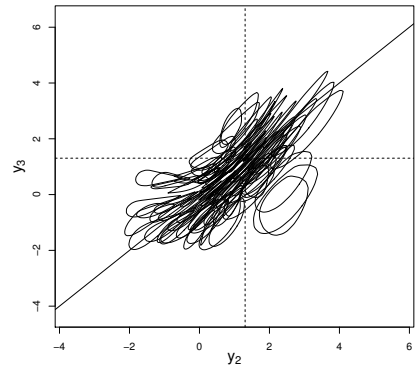

(b) $\left[\widetilde{\Lambda}\left(s_{i}, \boldsymbol{u}_{i}\right)\right]_{23}$
Fig. 6. Convex hulls of the two-dimensional projections of 100 focal sets $\widetilde{\Lambda}\left(s_{i}, \boldsymbol{u}_{i}\right)$ on the planes spanned by $\left(Y_{1}, Y_{2}\right)$ (left) and $\left(Y_{2}, Y_{3}\right)$ (right).

After $N$ focal sets $\widetilde{\Lambda}\left(s_{i}, \boldsymbol{u}_{i}\right), i=1, \ldots, N$ have been generated, they can be used to approximate the degrees of belief and plausibility of events of interest using (6). For instance, let us consider the following three events:

$$
\begin{aligned}
& E_{1}=\left(X_{T+1}>X_{T+2}>X_{T+3}\right) \\
& E_{2}=\left(X_{T+1}<0\right) \text { and }\left(X_{T+2}<0\right) \text { and }\left(X_{T+3}<0\right) \\
& E_{3}=\left(X_{T+1}>0\right) \text { and }\left(X_{T+2}>0\right) \text { and }\left(X_{T+3}>0\right) .
\end{aligned}
$$

Table I shows the degrees of belief and plausibility of these 
TABLE I

BELIEF, PLAUSIBILITY AND PROBABILITY OF THREE EVENTS. THE BELIEF-PLAUSIBILITY INTERVALS WERE COMPUTED BASED ON THE LIKELIHOOD-BASED AND CONFIDENCE BELIEF FUNCTIONS ON $\Theta$.

\begin{tabular}{cccccc} 
& \multicolumn{2}{c}{ Likelihood } & \multicolumn{3}{c}{ Confidence } \\
Event & $B e l$ & $P l$ & Bel & $P l$ & $P$ \\
\hline$E_{1}$ & 0.26 & 0.38 & 0.19 & 0.42 & 0.31 \\
$E_{2}$ & 0.024 & 0.073 & 0.011 & 0.11 & 0.086 \\
$E_{3}$ & 0.51 & 0.75 & 0.38 & 0.83 & 0.50 \\
\hline
\end{tabular}

TABLE II

ESTIMATED COVERAGE PROBABILITIES OF BELIEF-PLAUSIBILITY INTERVALS COMPUTED FROM THE LIKELIHOOD-BASED AND CONFIDENCE BELIEF FUNCTIONS ON $\theta$.

\begin{tabular}{cccc} 
& $E_{1}$ & $E_{2}$ & $E_{3}$ \\
\hline Likelihood & 0.987 & 0.924 & 0.782 \\
Confidence & 0.998 & 0.996 & 0.984 \\
\hline
\end{tabular}

three events, as well as their true conditional probability given $X_{T}=x_{T}$ estimated by Monte Carlo simulation. The beliefplausibility intervals were computed using both the likelihoodbased function and a 95\% confidence belief function on $\Theta$ (see Section III-A). We recall that, using the latter, we have, for at least $95 \%$ of the samples, $\operatorname{Bel}_{Y}(E) \leq P\left(E \mid X_{T}=x_{T}\right) \leq$ $\mathrm{Pl}_{Y}(E)$ for any event $E$ [12]. In contrast, these inequalities are not guaranteed to hold when the likelihood-based belief function is used. As shown in Table I, the belief-plausibility intervals computed from the confidence belief function do contain the true probabilities of the three events for this particular dataset, as expected. The likelihood-based intervals are narrower, but they do not have frequency-calibration properties.

To estimate the coverage probability of the beliefplausibility intervals (i.e., the probability of having $\operatorname{Bel}_{Y}(E) \leq P\left(E \mid X_{T}=x_{T}\right) \leq P l_{Y}(E)$, computed over a large number of realizations of the time series), we repeated the calculation with 1000 times series generated from the same distribution. The value of $x_{T}$ was fixed at 1.304641 , as in the previous calculations. The estimated coverage probabilities are reported in Table II. As expected, the belief-plausibility intervals based on the confidence belief function all have a coverage probability greater than $95 \%$, whereas the likelihood-based intervals have coverage probabilities below the nominal $95 \%$ level for events $E_{2}$ and $E_{3}$.

\section{CONCLUSIONS}

Although belief functions were initially introduced for statistical inference [3], they have not been widely used in statistical applications due to the difficulty to represent and manipulate continuous belief functions in $\mathbb{R}^{p}$. In this paper, we have proposed a method to represent and propagate continuous belief functions in multidimensional spaces based on the approximation of focal sets by point clouds. We have applied this method to the prediction of random vectors and, more specifically, to multistep time series prediction, extending the applicability of the prediction method introduced in [1]. The same method can be applied to a wide range of problems, such as, e.g., uncertainty propagation in numerical equations describing systems with uncertain parameters and/or inputs.

A key advantage of the point-cloud representation is the possibility to approximate any region of $\mathbb{R}^{p}$ with any accuracy, provided enough points are used. Furthermore, basic settheoretic operations such as projection, intersection, testing inclusion, etc., can be performed very efficiently. In contrast to the polygon representation recently introduced in [18], the point-cloud representation can be applied with any number $p$ of dimensions, but the curse of dimensionality obviously limits its use to spaces of moderate values of $p$, as the number of points needed to approximate a region with some accuracy grows exponentially with $p$. More simulations are needed to study the influence of $p$ and the number $M$ of points on approximation accuracy.

\section{REFERENCES}

[1] O. Kanjanatarakul, S. Sriboonchitta, and T. Denœux, "Forecasting using belief functions: an application to marketing econometrics," International Journal of Approximate Reasoning, vol. 55, no. 5, pp. 1113-1128, 2014.

[2] — - "Statistical estimation and prediction using belief functions: principles and application to some econometric models," International Journal of Approximate Reasoning, vol. 72, pp. 71-94, 2016.

[3] A. P. Dempster, "Upper and lower probabilities induced by a multivalued mapping," Annals of Mathematical Statistics, vol. 38, pp. 325-339, 1967.

[4] G. Shafer, A mathematical theory of evidence. Princeton, N.J.: Princeton University Press, 1976

[5] T. Denœux, "Likelihood-based belief function: justification and some extensions to low-quality data," International Journal of Approximate Reasoning, vol. 55, no. 7, pp. 1535-1547, 2014.

[6] H. T. Nguyen, "On random sets and belief functions," Journal of Mathematical Analysis and Applications, vol. 65, pp. 531-542, 1978.

[7] G. Shafer, "Allocations of probability," Annals of Probability, vol. 7, no. 5 , pp. 827-839, 1979.

[8] G. de Cooman and D. Aeyels, "A random set description of a possibility measure and its natural extension," IEEE Transactions on Systems, Man, and Cybernetics - Part A: Systems and Humans, vol. 30, no. 2, pp. 124$130,2000$.

[9] L. A. Zadeh, "Fuzzy sets as a basis for a theory of possibility," Fuzzy Sets and Systems, vol. 1, pp. 3-28, 1978.

[10] G. Shafer, "Dempster's rule of combination," International Journal of Approximate Reasoning, vol. 79, pp. 26-40, 2016.

[11] N. Wilson, "A Monte Carlo algorithm for Dempster-Shafer belief," in Proc. of the 7th Conference on Uncertainty in AI, B. D'Ambrosio, P. Smets, and P. Bonissone, Eds. Morgan Kaufmann, 1991.

[12] T. Denœux and S. Li, "Frequency-calibrated belief functions: Review and new insights," International Journal of Approximate Reasoning, vol. 92, pp. 232-254, 2018.

[13] Y. Pawitan, In all likelihood: Statistical Modelling and Inference Using Likelihood. Oxford: Clarendon Press, 2001.

[14] L. Sui, P. Feissel, and T. Denœux, "Identification of elastic properties in the belief function framework," International Journal of Approximate Reasoning, vol. 101, pp. 69-87, 2018.

[15] J. H. Halton, "Algorithm 247: Radical-inverse quasi-random point sequence," Communications of the ACM, vol. 7, no. 12, pp. 701-702, 1964.

[16] W. H. Greene, Econometric analysis, 7th ed. Upper Saddle River, NJ, USA: Prentice Hall, 2012.

[17] D. Machiwal and M. K. Jha, Hydrologic Time Series Analysis: Theory and Practice. Springer, 2012.

[18] N. Pellicanò, S. L. Hégarat-Mascle, and E. Aldea, "2CoBel: a scalable belief function representation for 2D discernment frames," International Journal of Approximate Reasoning, vol. 103, pp. 320-342, 2018. 